Article

\title{
Optimal System and New Approximate Solutions of a Generalized Ames's Equation
}

\author{
Marianna Ruggieri ${ }^{1}$ (D) and Maria Paola Speciale ${ }^{2, *(1)}$ \\ 1 Faculty of Engineering and Architecture, University of Enna "Kore", 94100 Enna, Italy; \\ marianna.ruggieri@unikore.it \\ 2 Department of Mathematics and Computer Science, Physical Sciences and Earth Science, \\ University of Messina, 98166 Messina, Italy \\ * Correspondence: mpspeciale@unime.it
}

Received: 30 July 2019; Accepted: 20 September 2019; Published: 1 October 2019

\begin{abstract}
In this paper, by applying Valenti's theory for the approximate symmetry, we introduce and define the concept of a one-dimensional optimal system of approximate subalgebras for a generalized Ames's equation; furthermore, the algebraic structure of the approximate Lie algebra is discussed. New approximately invariant solutions to the equation are found.
\end{abstract}

Keywords: partial differential equations; approximate symmetry and solutions

\section{Introduction}

In [1], Ames et al. performed the symmetries classification of the model

$$
u_{t t}=\left[f(u) u_{x}\right]_{x}
$$

that can describes the flow of one-dimensional gas, longitudinal wave propagation on a moving threadline, dynamics of a finite nonlinear string. The study of Equation (1) gave impetus to later investigations. In 2007, Bluman et al. [2] made an interesting nonlocal analysis of Equation (1). In this paper, our aim is to investigate the generalized model of Equation (1)

$$
u_{t t}=\left[f(u) u_{x}\right]_{x}+\varepsilon\left[\lambda(u) u_{t}\right]_{x x}
$$

where $f$ and $\lambda$ are smooth functions. $\varepsilon \ll 1$ is the small parameter for the perturbative analysis, while, when $\varepsilon=0$, we recover the unperturbed Equation (1).

As in the exact symmetries, even in the approximate one, an important task in determing approximately invariant solutions is to employ the concept of an optimal system of approximate subalgebras in order to obtain all the essentially different approximate invariant solutions.

In this manuscript, in the context of Valenti's theory [3], we define the definition of one-dimensional optimal system of approximate subalgebras for Equation (2). By its application, we get new approximate solutions for the generalized Ames's equation.

The plan of the manuscript is the following: in Section 2, after a brief introduction of the main concepts of Lie theory, we introduce the definition of Approximate Subalgebra and, finally, recall the main results of the approximate symmetry analysis of Equation (2). The Optimal Systems of one-dimensional approximate subalgebras are introduced and defined in Section 3 and the application of the method to the model is provided. Section 4 presents the reductions of Equation (2) to ordinary differential equations (ODEs) through the approximate optimal operators and new approximate solutions are obtained. Finally, in Section 5, the optimal system is used in order to 
construct new approximate non-invariant solutions for two other models linked to Equation (2) by a nonlocal transformation.

\section{On the Approximate Symmetry Classifications}

Various and different theories on approximate symmetries have been developed over the years; the first contribution on this argument is due to Baikov et al. [4], but the method does not consider an approximation in the perturbation meaning well, since, if we utilise the first order operator, the corresponding approximate solution could include higher order terms. Later, another interesting method was suggested by Fushchich et al. [5], where the strategy is consistent in the perturbation sense and produces correct terms for the approximate solutions, but it is impossible to work in hierarchy; then, the algebra could really grow. In 2004, Pakdemirli et al. [6] have compared these two methods. Afterwards, Valenti in [3] introduced his method, where, following the technique proposed in [5], he removes the "obstacle" of the impossibility of working in hierarchy, in accordance with the perturbation theory and, for this reason, we will apply this method.

Before entering in the details of approximate theory, we recall briefly the main concepts of Lie theory for a system of PDEs.

For a given system of differential equation

$$
\Delta\left(t, x, u, v, u^{(k)}, v^{(h)}\right)=0,
$$

where $t, x$ represents the independent variables. $u, v$ are the dependent variables and $u^{(k)}, v^{(h)}$ stand for all partial derivatives of $u$ and $v$ up to order $k$ and $h$, respectively.

The invertible transformations of $t, x, u, v$

$$
T=T(t, x, u, v, a), \quad X=X(t, x, u, v, a), \quad U=U(t, x, u, v, a), \quad V=V(t, x, u, v, a),
$$

depending on a continuous parameter $a$, are defined as one-parameter (a) (exact) Lie point symmetry transformations of Equation (3) if Equation (3) has the same form in the new variables $T, X, U, V$.

By expanding Equation (4) in Taylor's series around $a=0$, we obtain the infinitesimal transformations, according to the Lie theory:

$$
\begin{aligned}
& T=t+a \xi^{1}(t, x, u, v)+o\left(a^{2}\right), \quad X=x+a \xi^{2}(t, x, u, v)+o\left(a^{2}\right), \\
& U=u+a \eta^{1}(t, x, u, v)+o\left(a^{2}\right), \quad V=v+a \eta^{2}(t, x, u, v)+o\left(a^{2}\right),
\end{aligned}
$$

where their infinitesimals $\xi^{1}, \xi^{2}, \eta^{1}$ and $\eta^{2}$ are given by

$$
\xi^{1}(t, x, u, v)=\left.\frac{\partial T}{\partial a}\right|_{a=0}, \quad \xi^{2}(t, x, u, v)=\left.\frac{\partial X}{\partial a}\right|_{a=0}, \quad \eta^{1}(t, x, u, v)=\left.\frac{\partial U}{\partial a}\right|_{a=0}, \quad \eta^{2}(t, x, u, v)=\left.\frac{\partial V}{\partial a}\right|_{a=0} .
$$

The corresponding operator

$$
\Xi=\xi^{1}(t, x, u, v) \partial_{t}+\xi^{2}(t, x, u, v) \partial_{x}+\eta^{1}(t, x, u, v) \partial_{u}+\eta^{2}(t, x, u, v) \partial_{v}
$$

is known in the literature as the infinitesimal operator or generator of the Lie group.

The Lie group of point transformations, which leave a differential Equation (3) invariant, is obtained by means of the Lie's algorithm, with the requirement that the $\bar{k}$-order prolongation of Equation (7), which acts on Equation (3), is zero along the solutions, i.e.:

$$
\Xi^{\bar{k}} \Delta=\left.0\right|_{\Delta=0},
$$


where $\bar{k}=\max (k, h)$. The invariance condition Equation (8) produces an overdetermined system of linear differential equations (called determining equations) for the infinitesimals whose integration provides the generators of Lie Algebra admitted by Equation (3).

Now, we are able to define the concepts of Approximate Subalgebra of Equation (2).

Definition 1. We call Approximate Subalgebra of Equation (2) the (exact) subalgebra of the following system of PDEs:

$$
\begin{aligned}
L_{0}:= & u_{0 t t}-f\left(u_{0}\right) u_{0 x x}-f^{\prime}\left(u_{0}\right) u_{0 x}^{2}=0, \\
L_{1}:= & u_{1 t t}-f\left(u_{0}\right) u_{1 x x}-f^{\prime}\left(u_{0}\right) u_{0 x x} u_{1 x} \\
& -2 f^{\prime}\left(u_{0}\right) u_{0 x} u_{1 x}-f^{\prime \prime}\left(u_{0}\right) u_{0 x}^{2} u_{1} \\
& -\lambda^{\prime \prime}\left(u_{0}\right) u_{0 x}^{2} u_{0 t}-\lambda^{\prime}\left(u_{0}\right) u_{0 x x} u_{0 t} \\
& -2 \lambda^{\prime}\left(u_{0}\right) u_{0 x} u_{0 t x}-\lambda\left(u_{0}\right) u_{0 t x x}=0 .
\end{aligned}
$$

Systems (9)-(10) have been obtained considering $u(t, x, \varepsilon)$ analytics in $\varepsilon$ and expanded it in power series of $\varepsilon$, i.e.,

$$
u(t, x, \varepsilon)=u_{0}(t, x)+\varepsilon u_{1}(t, x)+\mathcal{O}\left(\varepsilon^{2}\right),
$$

where $u_{0}$ is the solution of the "unperturbed equation" (9) while $u_{1}$ can be obtained from the linear Equation (10).

For the sake of clarity, we briefly recall the main results of the symmetry classifications of Equation (2), which are obtained in [7].

The approximate generator of Equation (2) is written in the form

$$
\begin{aligned}
X= & \xi_{0}^{1}\left(t, x, u_{0}\right) \frac{\partial}{\partial t}+\xi_{0}^{2}\left(t, x, u_{0}\right) \frac{\partial}{\partial x}+\eta_{0}^{0}\left(t, x, u_{0}\right) \frac{\partial}{\partial u_{0}} \\
& +\left[\eta_{0}^{1}\left(t, x, u_{0}\right)+\eta_{1}^{1}\left(t, x, u_{0}\right) u_{1}\right] \frac{\partial}{\partial u_{1}}
\end{aligned}
$$

and the associate Approximate Principal Lie Algebra of Equation (2), obtained when $f\left(u_{0}\right)$ and $\lambda\left(u_{0}\right)$ are arbitrary functions of $u_{0}$, is

$$
X_{1}=\frac{\partial}{\partial t}, \quad X_{2}=\frac{\partial}{\partial x}, \quad X_{3}=t \frac{\partial}{\partial t}+x \frac{\partial}{\partial x}-u_{1} \frac{\partial}{\partial u_{1}},
$$

denoted with $L_{\mathcal{P}}^{\mathcal{A}}$. For suitable forms of functions $f\left(u_{0}\right)$ and $\lambda\left(u_{0}\right)$, we also get the symmetries summarized in the following Table 1 : 
Table 1. Classification of $f\left(u_{0}\right)$ and $\lambda\left(u_{0}\right)$ with the corresponding extensions of $L_{\mathcal{P}}^{\mathcal{A}}$ of Equation (2). $f_{0}, \lambda_{0}, p, q$ and $s$ are constitutive constants with $p \neq 0$.

\begin{tabular}{lll}
\hline Case & Forms of $f\left(u_{0}\right)$ and $\lambda\left(u_{0}\right)$ & Extensions of $\mathcal{A p p r o x}_{P}$ \\
\hline & $f\left(u_{0}\right)=f_{0} e^{\frac{1}{p} u_{0}}$ & $X_{4}=x \frac{\partial}{\partial x}+2 p \frac{\partial}{\partial u_{0}}+2 s u_{1} \frac{\partial}{\partial u_{1}}$ \\
\hline & $\lambda\left(u_{0}\right)=\lambda_{0} e^{\frac{1+s}{p} u_{0}}$ & \\
\hline$I I$ & $f\left(u_{0}\right)=f_{0}\left(u_{0}+q\right)^{\frac{1}{p}}$ & $X_{4}=x \frac{\partial}{\partial x}+2 p\left(u_{0}+q\right) \frac{\partial}{\partial u_{0}}+2 s u_{1} \frac{\partial}{\partial u_{1}}$ \\
& $\lambda\left(u_{0}\right)=\lambda_{0}\left(u_{0}+q\right)^{\frac{1+s}{p}-1}$ & \\
\hline III & $f\left(u_{0}\right)=f_{0}\left(u_{0}+q\right)^{-\frac{4}{3}}$ & $X_{4}=x \frac{\partial}{\partial x}-\frac{3}{2}\left(u_{0}+q\right) \frac{\partial}{\partial u_{0}}-\frac{3}{2} u_{1} \frac{\partial}{\partial u_{1}}$ \\
& $\lambda\left(u_{0}\right)=\lambda_{0}\left(u_{0}+q\right)^{-\frac{4}{3}}$ & $X_{5}=x^{2} \frac{\partial}{\partial x}-3 x\left(u_{0}+q\right) \frac{\partial}{\partial u_{0}}-3 x u_{1} \frac{\partial}{\partial u_{1}}$ \\
\hline
\end{tabular}

\section{Optimal System of Approximate Subalgebras}

In this section, we introduce the definition of Optimal Systems of one-dimensional approximate subalgebras for Equation (2), in the context of Valenti's theory of approximate symmetries; we begin by introducing the following definition:

Definition 2. We call the One-Dimensional Optimal system of Approximate Subalgebras of Equation (2) the one-dimensional, Optimal system of (exact) Subalgebras of systems (9)-(10).

As in the classical symmetries, even in the approximate one, an important instrument to get approximate solutions is to find the optimal system of approximate subalgebras in order to obtain all the essentially different approximate invariant solutions. In order to obtain reductions and to construct classes of group-invariant approximate solutions for Equation (2) in a systematic way, we will get an optimal system of one-dimensional approximate subalgebras for Equation (2).

Following [8] by using both the adjoint table [9] and the global matrix [10], we get one dimensional optimal system of subalgebras. We present only the details for the approximate principal Lie Algebra $L_{\mathcal{P}}$ of Equation (2). Thus, as a basis of $L_{\mathcal{P}}^{\mathcal{A}}$, we take adjoint operators, namely

$$
\mathcal{A}_{i}=-\left[X_{i}, X_{j}\right] \frac{\partial}{\partial X_{j}}, \quad(i, j=1,2,3),
$$

where, if $X_{i}$ and $X_{j}$ are vector fields, then their Lie bracket $\left[X_{i}, X_{j}\right]$ is the unique vector field satisfying

$$
\left[X_{i}, X_{j}\right]=X_{i}\left(X_{j}\right)-X_{j}\left(X_{i}\right)
$$

The commutator table of Approximate Principal Lie Algebra of case (13) is shown in Table 2.

Table 2. Commutator Table of the Approximate Lie Algebra of Equation (2). The $(i, j)$-th entry indicates $\left[X_{i}, X_{j}\right]=X_{i}\left(X_{j}\right)-X_{j}\left(X_{i}\right)$.

\begin{tabular}{cccc}
\hline & $X_{\mathbf{1}}$ & $\boldsymbol{X}_{\mathbf{2}}$ & $\boldsymbol{X}_{\mathbf{3}}$ \\
\hline$X_{1}$ & 0 & 0 & $X_{1}$ \\
\hline$X_{2}$ & 0 & 0 & $X_{2}$ \\
\hline$X_{3}$ & $-X_{1}$ & $-X_{2}$ & 0 \\
\hline
\end{tabular}


The adjoint representation can be denoted as $A d\left(\exp \left(\varepsilon_{i} X_{i}\right)\right) X_{j}$ and is written by summing the Lie series

$$
A d\left(\exp \left(\varepsilon_{i} X_{i}\right)\right) X_{j}=\sum_{n=0}^{+\infty} \frac{\varepsilon^{n}}{n !}\left(\operatorname{ad} X_{i}\right)^{n}\left(X_{j}\right)=X_{j}-\varepsilon\left[X_{i}, X_{j}\right]+\frac{\varepsilon^{2}}{2}\left[X_{i},\left[X_{i}, X_{j}\right]\right]-\ldots
$$

The Adjoint Table of Approximate Principal Lie Algebra spanned by operators (13) is written in Table 3:

Table 3. Adjoint Table of the Approximate Lie Algebra $L_{\mathcal{P}}$ of Equation (2). The $(i, j)$-th entry indicates $\operatorname{Ad}\left(\exp \left(\varepsilon_{i} X_{i}\right)\right) X_{j}=X_{j}-\varepsilon\left[X_{i}, X_{j}\right]+\frac{\varepsilon^{2}}{2}\left[X_{i},\left[X_{i}, X_{j}\right]\right]-\ldots$

\begin{tabular}{cccc}
\hline Ad & $X_{1}$ & $X_{2}$ & $X_{3}$ \\
\hline$X_{1}$ & $X_{1}$ & $X_{2}$ & $X_{3}-\varepsilon_{1} X_{1}$ \\
\hline$X_{2}$ & $X_{1}$ & $X_{2}$ & $X_{3}-\varepsilon_{2} X_{2}$ \\
\hline$X_{3}$ & $X_{1}\left(1+\varepsilon_{3}\right)$ & $X_{2}\left(1+\varepsilon_{3}\right)$ & $X_{3}$ \\
\hline
\end{tabular}

According the method given in [8], keeping in consideration the commutators, in the first line of Table 2, we get for instance

$$
\mathcal{A}_{1}=-X_{1} \frac{\partial}{\partial X_{3}}
$$

which generates the linear transformations

$$
X_{1}^{\prime}=X_{1}, \quad X_{2}^{\prime}=X_{2}, \quad X_{3}^{\prime}=-\varepsilon_{1} X_{1}+X_{3}
$$

Moreover, the linear transformations generated by each $\mathcal{A}_{i}$ can be obtained simply by checking the first, second, etc ... row of the Adjoint table (Table 3) of approximate principal Lie algebra $L_{\mathcal{P}}$ of Equation (2); for example, in the case of (16), the linear transformation is represented by the matrix

$$
M_{1}\left(\varepsilon_{1}\right)=\left(\begin{array}{ccc}
1 & 0 & 0 \\
0 & 1 & 0 \\
-\varepsilon_{1} & 0 & 1
\end{array}\right)
$$

Following [9], the global matrix $M$ of the adjoint transformations is the product of matrices $M_{i}\left(\varepsilon_{i}\right)$ associated with each $\mathcal{A}_{i}$. For $\mathcal{L}_{\mathcal{P}}$ of Equation (2), we have

$$
M=\Pi_{i=1}^{3} M_{i}\left(\varepsilon_{i}\right)=\left(\begin{array}{ccc}
1+\varepsilon_{3} & 0 & 0 \\
0 & 1+\varepsilon_{3} & 0 \\
-\varepsilon_{1}\left(1+\varepsilon_{3}\right) & -\varepsilon_{2}\left(1+\varepsilon_{3}\right) & 1
\end{array}\right)
$$

In order to obtain the global action of operators $\mathcal{A}_{i},(i=1, \ldots, 3)$, we apply the matrix $M^{T}$, transposed matrix of $M$, to an element of $L_{\mathcal{P}}$, i.e., $X_{o}=\sum_{i=1}^{3} a_{i} X_{i}$. Actually, it is preferable to work with the vector $\mathbf{a} \equiv\left(a_{1}, a_{2}, a_{3}\right)$; the coordinates of the transformed vector of a are

$$
\begin{aligned}
& \bar{a}_{1}=\left(1+\varepsilon_{3}\right) a_{1}-\varepsilon_{1}\left(1+\varepsilon_{3}\right) a_{3}, \\
& \bar{a}_{2}=\left(1+\varepsilon_{3}\right) a_{2}-\varepsilon_{2}\left(1+\varepsilon_{3}\right) a_{3}, \\
& \bar{a}_{3}=a_{3},
\end{aligned}
$$

and firstly we underline that these transformations leave invariant the component $a_{3}$ and provide the adjoint group $G^{\mathcal{A}}$ of $L_{\mathcal{P}}^{\mathcal{A}}$.

We can determine the optimal system of $L_{\mathcal{P}}$ by using a simple approach. We simplify any given vector $\mathbf{a} \equiv\left(a_{1}, a_{2}, a_{3}\right)$ through the above transformations. Then, distinguish the obtained vectors 
into nonequivalent classes, where we choose the one with the simplest form by which we obtain the following non-trivial operator of the optimal system of $L_{\mathcal{P}}$ :

$$
X_{o 1}=c_{1} X_{1}+c_{2} X_{2}+X_{3}=\left(c_{1}+t\right) \frac{\partial}{\partial t}+\left(c_{2}+x\right) \frac{\partial}{\partial x}-u_{1} \frac{\partial}{\partial u_{1}},
$$

where $c_{1}, c_{2}$ are real parameters.

Finally, starting from Case III of Table 1, we are able to construct the corresponding extensions of the optimal system of approximate subalgebras $\mathcal{L}_{p}$ of Case III for Equation (2), by using the Adjoint Table in the Appendix A (Table A1), which in this case reads as:

$$
\begin{aligned}
& X_{o 2}=c_{1} X_{1}+X_{3}+X_{4}=\left(c_{1}+t\right) \frac{\partial}{\partial t}+2 x \frac{\partial}{\partial x}-\frac{3}{2}\left(u_{0}+q\right) \frac{\partial}{\partial u_{0}}-\frac{5}{2} u_{1} \frac{\partial}{\partial u_{1}}, \\
& X_{03}=c_{1} X_{1}+c_{2} X_{2}+X_{5}=c_{1} \frac{\partial}{\partial t}+\left(c_{2}+x^{2}\right) \frac{\partial}{\partial x}-3 x\left(u_{0}+q\right) \frac{\partial}{\partial u_{0}}-3 x u_{1} \frac{\partial}{\partial u_{1}} .
\end{aligned}
$$

\section{Reduction to ODEs and New Approximate Invariant Solution for the Generalized Ames's Equation}

Based on the results obtained in the previous section, thanks to the approximate generators, we can construct the corresponding reduced ODEs of Equation (2); indeed, as we know from literature, group classification problems are interesting not only from a purely mathematical point of view but above all in the applications that can be achieved [9-12].

We obtain an approximate solution for Equation (2) considering the approximate operator (17) of $\mathcal{L}_{p}$, i.e.,

$$
X_{o 1}=c_{1} X_{1}+c_{2} X_{2}+X_{3}=\left(c_{1}+t\right) \frac{\partial}{\partial t}+\left(c_{2}+x\right) \frac{\partial}{\partial x}-u_{1} \frac{\partial}{\partial u_{1}},
$$

we get the following transformation

$$
z=\frac{c_{2}+x}{c_{1}+t}, \quad u_{0}=\phi(z), \quad u_{1}=\frac{\psi(z)}{c_{1}+t^{\prime}}
$$

which maps Equation (2) into the following ODEs:

$$
\begin{aligned}
& 2 z \phi^{\prime}-f^{\prime} \phi^{\prime 2}+\left(z^{2}-f\right) \phi^{\prime \prime}=0 \\
& \lambda z \phi^{\prime \prime \prime}-\left(f^{\prime} \psi-2 \lambda-3 z \lambda^{\prime} \phi^{\prime}\right) \phi^{\prime \prime}+\left(z^{2}-f\right) \psi^{\prime \prime} \\
& \quad+\left(z \lambda^{\prime \prime} \phi^{\prime}+2\left(\lambda^{\prime}-f^{\prime \prime}\right) \psi\right) \phi^{\prime \prime}-2\left(f^{\prime} \phi^{\prime}-2 z\right) \psi^{\prime}+2 \psi=0
\end{aligned}
$$

$f=f(u)$ and $\lambda=\lambda(u)$ being arbitrary functions of its argument. When $f=f_{0}=$ constant and $\lambda=\lambda_{0}=$ constant, by integration of the reduced equations, we get

$$
\phi=k_{1}+\frac{k_{2}}{f_{0}} \operatorname{arctanh}\left(\frac{z}{\sqrt{f_{0}}}\right), \quad \psi=\frac{\left(k_{3}+k_{4} z\right)\left(f_{0}-z^{2}\right)+k_{2} \lambda_{0} z}{\left(f_{0}-z^{2}\right)^{2}},
$$

where $k_{i},(i=1, \ldots, 4)$ are arbitrary constants, and, as a consequence, we obtain the following solution of Equation (2)

$$
\begin{aligned}
u= & k_{1}+\frac{k_{2}}{f_{0}} \operatorname{arctanh}\left(\frac{c_{2}+x}{\sqrt{f_{0}}\left(c_{1}+t\right)}\right) \\
& +\varepsilon \frac{\left(k_{3}\left(c_{1}+t\right)+k_{4}\left(c_{2}+x\right)\right)\left(f_{0}\left(c_{1}+t\right)^{2}-\left(c_{2}+x\right)^{2}\right)+k_{2} \lambda_{0}\left(c_{1}+t\right)^{2}\left(c_{2}+x\right)}{\left(f_{0}\left(c_{1}+t\right)^{2}-\left(c_{2}+x\right)^{2}\right)^{2}} .
\end{aligned}
$$


Another solution can be obtained by the operator

$$
X_{o 2}=\left(c_{1}+t\right) \frac{\partial}{\partial t}+2 x \frac{\partial}{\partial x}-\frac{3}{2}\left(u_{0}+q\right) \frac{\partial}{\partial u_{0}}-\frac{5}{2} u_{1} \frac{\partial}{\partial u_{1}}
$$

when $f=f_{0}\left(u_{0}+q\right)^{-4 / 3}$ and $\lambda=\lambda_{0}\left(u_{0}+q\right)^{-4 / 3}$ (Case III of Table 1), which leads to get the following transformation of variables:

$$
z=\frac{x}{\left(c_{1}+t\right)^{2}}, \quad u_{0}=\frac{\phi(\xi)}{\left(c_{1}+t\right)^{3 / 2}}-q, \quad u_{1}=\frac{\psi(\xi)}{\left(c_{1}+t\right)^{5 / 2}},
$$

and to the following reduced equations:

$$
\begin{aligned}
& 12\left(4 z^{2} \phi^{\frac{4}{3}}-f_{0}\right) \phi \phi^{\prime \prime}+16\left(f_{0} \phi^{\prime}+9 z \phi^{\frac{7}{3}}\right) \phi^{\prime}+45 \phi^{\frac{10}{3}}=0 \\
& 72 \lambda_{0} z \phi^{2} \phi^{\prime \prime \prime}-6 \phi\left(48 \lambda_{0} z \phi^{\prime}-21 \lambda_{0} \phi-8 f_{0} \psi\right) \phi^{\prime \prime}+36\left(4 z^{2} \phi^{\frac{4}{3}}-f_{0}\right) \phi^{2} \psi^{\prime \prime} \\
& \quad+96\left(f_{0} \phi^{\prime}+6 z \phi^{\frac{7}{3}}\right) \phi \psi^{\prime}+56\left(4 \lambda_{0} z \phi^{\prime}-2 f_{0} \psi-21 \lambda_{0} \phi\right) \phi^{2}+315 \phi^{\frac{10}{3}} \psi=0
\end{aligned}
$$

that admit as a solution

$$
\phi=\left(\frac{\sqrt{f_{0}}}{z}\right)^{3 / 2}, \quad \psi=\frac{1}{z^{3 / 2}}\left(k_{1}+\frac{3}{2} k_{2} \log z-\lambda_{0}\left(\frac{\log z}{4 \sqrt{f_{0}}}\right)^{2}\right)
$$

$k_{1}$ and $k_{2}$ being arbitrary constants. Thus, a solution of (2) is

$$
u=\sqrt{\frac{c_{1}+t}{x^{3}}}\left(f_{0}^{3 / 4}\left(c_{1}+t\right)-q+\varepsilon\left(k_{1}+\frac{3}{2} k_{2} \log \frac{x}{\left(c_{1}+t\right)^{2}}-\lambda_{0}\left(\frac{\log \frac{x}{\left(c_{1}+t\right)^{2}}}{4 \sqrt{f_{0}}}\right)^{2}\right)\right) .
$$

Finally, if we consider the approximate operator:

$$
X_{o 3}=c_{1} X_{1}+c_{2} X_{2}+X_{5}=c_{1} \frac{\partial}{\partial t}+\left(c_{2}+x^{2}\right) \frac{\partial}{\partial x}-3 x\left(u_{0}+q\right) \frac{\partial}{\partial u_{0}}-3 x u_{1} \frac{\partial}{\partial u_{1}},
$$

we get the following transformation:

$$
z=\frac{1}{\sqrt{c_{2}}} \arctan \left(\frac{c_{1}}{c_{2}} x\right)-\frac{t}{c_{1}}, \quad u_{0}=\left(\frac{c_{2}}{c_{2}+c_{1} x^{2}}\right)^{\frac{3}{2}} \phi(z)-q, \quad u_{1}=\left(\frac{c_{2}}{c_{2}+c_{1} x^{2}}\right)^{\frac{3}{2}} \psi(z),
$$

which maps Equation (2) into the following ODEs:

$$
\begin{aligned}
& 3\left(c_{2}^{2} \phi^{\frac{4}{3}}-c_{1}^{4} f_{0}\right) \phi \phi^{\prime \prime}+c_{1}^{4} f_{0}\left(4 \phi^{2}+9 c_{2} \phi^{2}\right)=0, \\
& 9 c_{1}^{3} \lambda_{0} \phi^{2} \phi^{\prime \prime \prime}+12 \phi\left(c_{1} f_{0} \psi-3 \lambda_{0} \phi^{\prime}\right) \phi^{\prime \prime}+9\left(c_{2}^{2} \phi^{\frac{4}{3}}-c_{1}^{4} f_{0}\right) \phi^{2} \psi^{\prime \prime} \\
& \quad+24 c_{1}^{4} f_{0} \phi \phi^{\prime} \psi^{\prime}-c_{1}^{3}\left(c_{1} f_{0} \psi-\lambda_{0} \phi^{\prime}\right)\left(9 c_{2} \phi^{2}+28 \phi^{\prime 2}\right)=0 .
\end{aligned}
$$

A particular solution is given by $\phi=0$, so that the solution of Equation (2) is

$$
u=-q+\varepsilon\left(\frac{c_{2}}{c_{2}+c_{1} x^{2}}\right)^{\frac{3}{2}} \psi(z)
$$

with $\psi(z)$ arbitrary function of

$$
z=\frac{1}{\sqrt{c_{2}}} \arctan \left(\frac{c_{1}}{c_{2}} x\right)-\frac{t}{c_{1}}
$$


A simple solution that can be obtained from (24) by setting $c_{1}=c_{2}=0$; the similarity solution and the variables become, respectively,

$$
z=t, \quad u_{0}=x^{-3} \phi(t)-q, \quad u_{1}=x^{-3} \psi(t),
$$

while the corresponding reduced ODEs of (2) assume the simple form:

$$
\phi^{\prime \prime} \phi^{\frac{1}{3}}=0, \quad \psi^{\prime \prime} \phi^{\frac{4}{3}}=0,
$$

which admit the solution

$$
\phi=h_{1} t+h_{0}, \quad \psi=k_{1} t+k_{0}
$$

with $h_{1}, h_{0}, k_{1}$ and $k_{0}$ arbitrary constants of integration. Then, we can write the invariant approximate solution for Equation (2)

$$
u(t, x)=\frac{h_{1} t+h_{0}}{x^{3}}-q+\varepsilon \frac{k_{1} t+k_{0}}{x^{3}}+\mathcal{O}\left(\varepsilon^{2}\right) .
$$

\section{The Potential System Associated with the Generalized Ames's Equation}

We recall that, for any given system of PDEs, it is possible to construct nonlocally related potential systems that have the same solutions of the given system [2]. The transformation that allows for mapping the given system of PDEs into nonlocally potential systems is defined "nonlocal transformation".

Thus, we have that Equation (2) leads to getting the following system:

$$
\begin{aligned}
& u_{t}-v_{x}=0, \\
& v_{t}-\left(\int^{u} f(s) d s+\varepsilon \lambda(u) v_{x}\right)_{x}=0,
\end{aligned}
$$

studied in [13], which can be treated as the potential system of Equation (2).

In addition, if we consider the nonlocal transformation $u=w_{x}, v=w_{t}$, systems (32)-(33) are equivalent to

$$
w_{t t}=f\left(w_{x}\right) w_{x x}+\varepsilon\left[\lambda\left(w_{x}\right) w_{t x}\right]_{x}
$$

studied in [8,14]. Special cases of models belonging to the class of Equation (34) can be found in [15-19]. Furthermore, some questions related to global existence, uniqueness and stability of solutions have been addressed in [20,21].

In Ref. [7], the authors have proved a Theorem affirming that For any $f$ and $\lambda$, an approximate symmetry admitted by the systems (32)-(33) and Equation (34) defines an approximate symmetry admitted by Equation (2); conversely, some approximate symmetries of Equation (2) do not induce approximate symmetries of Equation (34) and the systems (32)-(33).

In a few words, the classification of Equation (2) is richer than those of systems (32)-(33) and of Equation (34). In fact, while there is a correspondence between the approximate symmetry operators $X_{1}, \ldots, X_{4}$ admitted by Equation (2) with the ones admitted by (34), the operator $X_{5}$ reported in case III of Table 2 is a new approximate operator, admitted only by Equation (2).

According to this theorem, in light of the determination of the one-dimensional optimal system of approximate subalgebras, we are able to get approximate invariant and non-invariant solutions for the systems (32)-(33) and Equation (34).

For better clarification: the correspondence between the approximate symmetry operators $X_{1}, \ldots$, $X_{4}$ admitted by Equation (2) with the ones admitted by (34), allows us to get approximate invariant solutions for the systems (32)-(33) and Equation (34); for instance, the general solution (21) which we have obtained in this paper, when $k_{2}=0$, includes the solution obtained considering an approximate 
operator admitted by Equation (34) and found in [8]. Instead, starting from the approximate solution (31), obtained by means of the operator $X_{03}$ that involves the generator $X_{5}$, we are able to obtain approximate non-invariant solutions for the systems (32)-(33) and Equation (34) that could not have been obtained from the symmetry analysis and reductions performed in $[13,14]$ because the operator $X_{5}$ is new and it is admitted only by Equation (2).

New Approximate Non-Invariant Solutions for Equation (35) and the Potential System

Starting from the approximate solution (31), keeping in consideration the nonlocal transformation $u=w_{x}$, by integrating (31) with respect to $x$, we obtain:

$$
w(t, x)=-\frac{h_{1} t+h_{0}}{2 x^{2}}-q x+\chi_{0}(t)+\varepsilon\left(\frac{k_{1} t+k_{0}}{2 x^{2}}+\chi_{1}(t)\right),
$$

where $\chi_{0}(t)$ and $\chi_{1}(t)$, at this stage, are arbitrary functions of $t$. When we substitute them into (34), we get that it must be linear in their arguments, so we obtain the following new non-invariant approximate solution for Equation (34):

$$
\begin{aligned}
& w(t, x)=-\frac{h_{1} t+h_{0}}{2 x^{2}}-q x-\frac{27 f_{0}}{10 h_{1}^{2}}\left(h_{1} t+h_{0}\right)^{\frac{5}{3}}+K_{1} t \\
& -\varepsilon\left[\frac{k_{1} t+k_{0}}{2 x^{2}}-\frac{9\left(h_{0}+h_{1} t\right)^{\frac{2}{3}}}{10 h_{1}^{3}}\left[45 \lambda_{0} h_{1}^{2}+f_{0}\left(h_{1} k_{1} t-5 h_{1} k_{0}+6 h_{0} k_{1}\right)\right]-K_{2} t\right]+\mathcal{O}\left(\varepsilon^{2}\right),
\end{aligned}
$$

where $K_{1}$ and $K_{2}$ are arbitrary constants.

Finally, the non-invariant approximate solution for the system (32)-(33) reads as

$$
\begin{aligned}
& u=\frac{h_{1} t+h_{0}}{x^{3}}-q+\varepsilon \frac{k_{1} t+k_{0}}{x^{3}}, \\
& v=-\frac{h_{1}}{2 x^{2}}-\frac{9 f_{0}}{2 h_{1}}\left(h_{1} t+h_{0}\right)^{\frac{2}{3}}+K_{1} \\
& -\varepsilon\left[\frac{k_{1} t+k_{0}}{2 x^{2}}-\frac{9\left(h_{0}+h_{1} t\right)^{\frac{2}{3}}}{10 h_{1}^{3}}\left[45 \lambda_{0} h_{1}^{2}+f_{0}\left(h_{1} k_{1} t-5 h_{1} k_{0}+6 h_{0} k_{1}\right)\right]-K_{2} t\right] .
\end{aligned}
$$

\section{Conclusions}

In this manuscript, we worked following the method of Valenti's approximate symmetries; moreover, thanks to the link between the approximate symmetries of the three related models (2), (32)-(33), (34) and the definition of one-dimensional optimal system of approximate subalgebras, we are also capable in this manuscript to get new approximate, invariant and non-invariant, solutions not only for the generalized Ames's Equation (2), but also for Equation (34) and the systems (32)-(33) which could not be obtained from the approximate symmetry analysis performed in $[13,14]$.

Author Contributions: M.R. and M.P.S. worked together in the derivation of the mathematical results. Both authors provided critical feedback and helped shape the research, analysis and manuscript.

Funding: This research received no external funding.

Acknowledgments: The authors acknowledge the support by G.N.F.M. of INdAM.

Conflicts of Interest: The authors declare no conflict of interest. 


\section{Appendix A}

In this Appendix, the Adjoint Table (Table A1) of the case III is reported.

Table A1. Adjoint Table of the Approximate Lie Algebra of Equation (2).

\begin{tabular}{cccccc}
\hline Ad & $X_{\mathbf{1}}$ & $X_{\mathbf{2}}$ & $X_{3}$ & $X_{\mathbf{4}}$ & $X_{5}$ \\
\hline$X_{1}$ & $X_{1}$ & $X_{2}$ & $X_{3}-\varepsilon_{1} X_{1}$ & $X_{4}$ & $X_{5}$ \\
\hline$X_{2}$ & $X_{1}$ & $X_{2}$ & $X_{3}-\varepsilon_{2} X_{2}$ & $X_{4}-\varepsilon_{2} X_{2}$ & $X_{5}-2 \varepsilon_{2} X_{4}$ \\
\hline$X_{3}$ & $X_{1}\left(1+\varepsilon_{3}\right)$ & $X_{2}\left(1+\varepsilon_{3}\right)$ & $X_{3}$ & $X_{4}$ & $X_{5}\left(1-\varepsilon_{3}\right)$ \\
\hline$X_{4}$ & $X_{1}$ & $X_{2}\left(1+\varepsilon_{4}\right)$ & $X_{3}$ & $X_{4}$ & $X_{5}\left(1-\varepsilon_{4}\right)$ \\
\hline$X_{5}$ & $X_{1}$ & $X_{2}+2 \varepsilon_{5} X_{4}$ & $X_{3}+\varepsilon_{5} X_{5}$ & $X_{4}+\varepsilon_{5} X_{5}$ & $X_{5}$ \\
\hline
\end{tabular}

\section{References}

1. Ames, W.F.; Lohner, R.J.; Adams, E. Group properties of $u_{t t}=\left[f(u) u_{x}\right]_{x}$. Int. J. Non-Linear Mech. 1981, $16,439-447$. [CrossRef]

2. Bluman, G.; Cheviakov, A.F. Nonlocally related systems, linearization and nonlocal symmetries for the nonlinear wave equation. J. Math. Anal. Appl. 2007, 333, 93-111. [CrossRef]

3. Valenti, A. Approximate symmetries for a model describing dissipative media. In Proceedings of the MOGRAN X, Larnaca, Cyprus, 24-31 October 2004; pp. 236-242.

4. Bă̌kov, V.A.; Gazizov, R.K.; Ibragimov, N.H. Approximate Symmetries. Math. USSR Sb. 1989, 64, 427-441. [CrossRef]

5. Fushchich, W.I.; Shtelen, W.M. On approximate symmetry and approximate solutions of the nonlinear wave equation with a small parameter. J. Phys. A Math. Gen. 1989, 22, 887-890. [CrossRef]

6. Pakdemirli, M.; Yurusoy, M.; Dolapc, I. Comparison of approximate symmetry methods for differential equation. Acta Appl. Math. 2004, 80, 243-271. [CrossRef]

7. Ruggieri, M.; Speciale, M.P. Lie group analysis of a wave equation with a small nonlinear dissipation. Ric. Di Mat. 2017, 66, 27-34. [CrossRef]

8. Ruggieri, M.; Speciale, M.P. Optimal System and Approximate Solutions of Nonlinear Dissipative Media. Math. Methods Appl. Sci. 2019. [CrossRef]

9. Olver, P.J. Applications of Lie Groups to Differential Equations; Springer: New York, NY, USA, 1986.

10. Ovsiannikov, L.V. Group Analysis of Differential Equations; Academic Press: New York, NY, USA, 1982.

11. Ibragimov, N.H. CRC Hanbook of Lie Group Analysis of Differential Equations; CRC Press: Boca Raton, FL, USA, 1994.

12. Ruggieri, M.; Speciale, M.P. Quasi Self-adjoint Coupled KdV-like Equations. AIP Conf. Proc. 2013, 1558, 1220-1223.

13. Ruggieri, M.; Speciale, M.P. Approximate symmetries in viscoelasticity. Theor. Math. Phys. 2016, 189,1500-1508. [CrossRef]

14. Ruggieri, M.; Valenti A. Approximate symmetries in nonlinear viscoelastic media. Bound. Value Probl. 2013, 2013, 143. [CrossRef]

15. Ruggieri, M.; Speciale, M.P. Approximate Analysis of a Nonlinear Dissipative Model. Acta Appl. Math. 2014, 132, 549-559. [CrossRef]

16. Valenti, A. Approximate symmetries of a viscoelastic model. In Proceedings of the WASCOM 2007 Baia Samuele, Sicily, Italy, 30 June-7 July 2008; Manganaro, N., Monaco, R., Rionero, S., Eds.; World Science Publishing: Singapore, 2008; pp. 582-588.

17. Ruggieri, M.; Valenti, A. Symmetries and reduction techniques for dissipative models. J. Math. Phys. 2009, 50, 063506. [CrossRef]

18. Ruggieri, M.; Valenti, A. Exact solutions for a nonlinear model of dissipative media. J. Math. Phys. 2011, 52, 043520. [CrossRef]

19. Ruggieri, M. Kink solutions for a class of generalized dissipative equation. Abstr. Appl. Anal. 2012. [CrossRef] 
20. Dafermos, C.M. The mixed initial-boundary value problem for the equations of nonlinear one-dimensional viscoelasticity. J. Differ. Equ. 1969, 6, 71-86. [CrossRef]

21. MacCamy, R.C. Existence, uniqueness and stability of $u_{t t}=\frac{\partial}{\partial x}\left[\sigma\left(u_{x}\right)+\lambda\left(u_{x}\right) u_{x t}\right]$. Indiana Univ. Math. J. 1970, 20, 231-238. [CrossRef]

(C) 2019 by the authors. Licensee MDPI, Basel, Switzerland. This article is an open access article distributed under the terms and conditions of the Creative Commons Attribution (CC BY) license (http:// creativecommons.org/licenses/by/4.0/). 